\title{
Brain Structure Visualization using Spectral Fiber Clustering
}

\author{
Jan Klein * \\ MeVis, Germany \\ Felix Ritter \\ MeVis, Germany
}

\author{
Horst K. Hahn \\ MeVis, Germany
}

\author{
Jan Rexilius \\ MeVis, Germany
}

\author{
Heinz-Otto Peitgen \\ MeVis, Germany
}

\begin{abstract}
The visualization of human brain structure is an important new challenge in the area of computer graphics. We present a novel algorithm that allows for visualizing white matter fiber tracts in realtime. Related structure is grouped by our spectral clustering method very efficiently and curvature and spatial depth of the fibers are accentuated by new GPU-based illustrative rendering techniques. Our fiber clustering results are much more accurate and comprehensible than that obtained by previous feature space methods.
\end{abstract}

\section{Introduction}

An explicit geometrical reconstruction of white matter fiber tracts has become a hot topic in medical visualization [Ibanez et al. 2005]. Fiber clustering offers an opportunity for improving the perception of the fiber bundles and connectivity in the human brain by grouping anatomically similar or related fibers. In a first step, an affinity matrix representing the similarity is computed from which afterwards the fibers are grouped into clusters. However, due to the large number of 3D points constituting each single fiber, existing methods map the high dimensional fiber data to a low dimensional feature space [Enders et al. 2005]. Thus, geometrical information is neglected resulting in imprecise clusters. In addition, the visualization of spatial relations and the separation of adjacent fibers becomes difficult when color is used to encode spatial and non-spatial properties like local fiber direction and cluster indices.

\section{Grid-Based Spectral Clustering}

Examining the anatomical structure of white matter fiber tracts, we observed that several non-convex clusters have to be detected for a plausible visualization. Thus, we propose to use spectral clustering algorithms that already have become popular in fields like image segmentation or processing of point-sampled geometry due to their suitability for non-convexity [Pauly and Gross 2001].

Using the affinity matrix, our spectral algorithm first determines its eigenvalues and eigenvectors, that, after a transformation, can easily be clustered by any hierarchical clustering method. The number of clusters is automatically determined by our new eigenvalue regression. The idea is to perform a linear regression of the eigenvalues to find the largest difference between two consecutive eigenvalues in a list of descending order, because the relevant eigenvalues for nearly separated clusters are bordered by an eigengap.

In order to gather more accurate spatial information of the fibers than using the feature space method, we utilize a space partitioning grid so that each fiber point is assigned with a constant weight to the cell containing it. Moreover, each point is additionally assigned to the surrounding cells. The corresponding weights are reduced by a factor which takes the distance from the central cell and the number of cells at the same distance into account. This allows for a more reliable clustering of fiber bundles with diameters exceeding a single cell. Let $w_{i}(x, y, z)$ denote the sum of all weights stored in cell $(x, y, z)$ arising from fiber $i$. The affinity matrix with entries $a_{i j}$ can now easily be calculated by

$$
a_{i j}:=\sum_{(x, y, z)} w_{i}(x, y, z) \cdot w_{j}(x, y, z) \quad i \neq j
$$

so that $a_{i j}$ is increased if fiber $i$ and $j$ contain data points that are assigned to the same cell.

\footnotetext{
*e-mail: klein@mevis.de
}

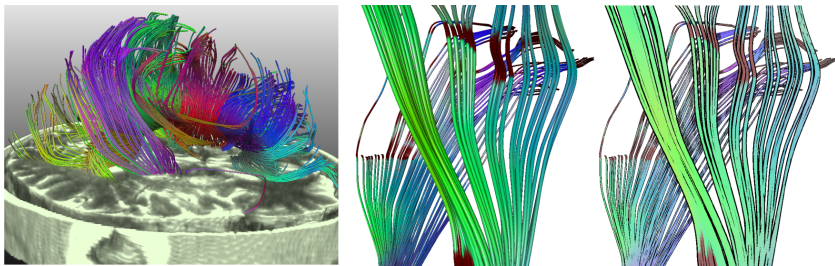

Figure 1: Left: fibers grouped by our spectral clustering, center: streamtubes color-coded to local direction, low fractional anisotropy is highlighted in red, right: illustrative rendering.

Considering the limited spatial fiber density, it is easy to show that the overall calculation of the affinity matrix is in $O(n)$ in the average case where $n$ denotes the number of fibers. This improves on feature space methods which need $O\left(n^{2}\right)$.

\section{Enhancing Spatial Perception}

For visualizing the fiber tracts, polygon-based streamtubes colored corresponding to the cluster indices can be used. We propose to perform alpha blending so that important medical information like fractional anisotropy can additionally be displayed (Fig. 1 left). However, if color is used to encode spatial and non-spatial information (Fig. 1 center), the visualization of spatial relations and the perceptive separation of adjacent fiber bundles becomes demanding, especially in the case of multi-parameter visualizations.

Therefore, we utilize and extend on non-photorealistic rendering techniques [Strothotte and Schlechtweg 2002] to emphasize spatial depth and topology with only limited use of color. A new GPU-based hatching algorithm as well as distance-encoded, strokeshadows improve the perception of spatial relations, such as curvature. Both algorithms rely on the extraction of depth differences in multi-renditions of the fibers to particularly enhance the perception of spatial depth. Only important surface features are accentuated thereby minimizing the occlusion of properties illustrated by colorcoding. Color may now be used as a distinct visual attribute for the visualization of non-spatial properties (Fig. 1 right).

\section{Results and Conclusion}

The proposed methods allow for an automatic and efficient visualization of white matter fiber tracts. Our spectral fiber clustering provides a better understanding and perception of the anatomy. We reduced the time for computing the affinity matrix from $O\left(n^{2}\right)$ to only $O(n)$ which makes it useful for real-time applications. As an alternative to our streamtube visualization, new algorithms for the spatially accentuated illustration of fibers have been developed so that color may be used to encode additional information.

\section{References}

Enders, F., Sauber, N., Merhof, D., Hastreiter, P., Nimsky, C., AND STAMMINGER, M. 2005. Visualization of white matter tracts with wrapped streamlines. In IEEE Visualization 2005, 51-58.

Ibanez, L., Aylward, S., And Kindlmann, G. 2005. Hot topics in 3D medical visualization. In SIGGRAPH 2005 Tutorial.

PAuly, M., AND GRoss, M. 2001. Spectral processing of point-sampled geometry. In SIGGRAPH 2001, 379-386.

Strothotte, T., And SchlechtweG, S. 2002. Non-Photorealistic Computer Graphics: Modeling, Rendering, and Animation. Morgan Kaufmann Publishers, San Francisco. 